\title{
Movilidad subjetiva, capabilities y educación ${ }^{1}$
}

Pedro Flores-Crespo ${ }^{2}$

Nadyra Rodríguez Arias ${ }^{3}$

Universidad Autónoma de Querétaro

\section{Artículo científico}

Material original autorizado para su primera publicación en el Journal de Ciencias Sociales, Revista Académica de la Facultad de Ciencias Sociales de la Universidad de Palermo.

Recepción: 17-02-2020

Aprobación: 06-10-2020

Resumen: Este artículo responde a la pregunta de cómo han cambiado ciertas percepciones a lo largo de la trayectoria de vida de seis egresados con el título de Técnico Superior Universitario de una universidad tecnológica de México y qué decisiones están tomando a partir de este cambio de subjetividad. Para ello, se discute la importancia de combinar variables subjetivas (percepción) con las de tipo objetivo (ingreso) en el estudio de la movilidad social. Se elige el enfoque de las capabilities (Sen, 1985, 1987, 1999, 2009) como el marco de análisis primordial dado su énfasis en la racionalidad individual amplia, así como en su aporte conceptual referido a la libertad sustantiva para elegir estilos de vida valiosos. Por medio de un estudio longitudinal de panel, se recaba información para dar cuenta de un patrón de movilidad subjetiva ascendente, la cual mantiene su relación con la de tipo objetivo,

\footnotetext{
${ }^{1}$ Este artículo se desprende de un proyecto de investigación más amplio, el cual, fue apoyado en un primer momento por la Secretaría de Educación Pública a través del programa Prodep y posteriormente por la Universidad Autónoma de Querétaro mediante el esquema (FOFIUAQ/FCP 2018/10). PFC agradece el espacio brindado por la Harvard Graduate School of Education (HGSE) para terminar este estudio durante la estancia sabática y en especial, a su anfitrión, el profesor Fernando Reimers.

2 Es investigador de la Universidad Autónoma de Querétaro en México. Ha sido profesor visitante en Harvard y en el Instituto sobre Educación Efectiva en York, Inglaterra. De 2007 a 2009, fue el coordinador de redes de la Human Development and Capability Association y promovió, junto con Mathias Nebel y Rodolfo de la Torre, el primer congreso sobre capabilities en América Latina. Escribe una columna semana en el periódico El Universal de Querétaro y disfruta dar clases de teoría social, implementación de políticas y desigualdad educativa. Correo electrónico: pedrofc71@yahoo.com

${ }^{3}$ Maestra en Economía por la Universidad de Guanajuato y Doctora en Ciencias Económico Administrativas con especialidad en Estudios Económicos por la Universidad Autónoma de Querétaro, en México. Áreas de investigación: pobreza, desigualdad, educación y movilidad social. Profesora en la Universidad Autónoma de Querétaro y en la Arkansas State University Campus Querétaro.

Correo electrónico: nadyra.rodriguez@uaq.mx
} 
tal como lo han documentado otros estudios. Además, se observa una relación positiva sobre sus percepciones acerca de la educación y sus logros. La manera en que los egresados de esta universidad perciben sus libertades y las de sus hijos lleva a pensar en una función social de la educación superior tecnológica que merece un mayor debate y además, demanda estudio con poblaciones más extensas para constatar los hallazgos aquí reportados.

Palabras clave: educación superior tecnológica; movilidad social subjetiva; capabilities, subjetividad; México.

\section{Subjective mobility, capabilities and education}

Abstract: How have the perceptions of a group of Mexican university graduates changed over time? And what kind of decisions could these graduates take when their subjectivity has been modified? This article addresses both questions by focusing in the life path of a group of associate professionals from one of the technological universities in Mexico. In doing so, the need of taking into account both subjective (perception) and objective (monetary income) variables in the study of social mobility is stressed. The capabilities approach (Sen, 1985, $1987,1999,2009)$ is applied because of the focus on individual rationality and the freedom an individual has for choosing what she/he values for her/his life achievements. A longitudinal study is designed to analyse the social mobility, and it is found an upward trend in subjective mobility among the participants, which is closely related to the observed objective mobility, according to what has been supported by previous studies. These findings argue that the group of university graduates have lifted their own expectations about education, therefore, their actions to support a new generation is potentially real, and due to the successful outcomes they have experienced, their perceptions of education are positively related to what they value the most. This may enhance the social function of the technological higher education in Mexico, but further analysis is required.

Keywords: technological higher education; subjective social mobility; capabilities, subjectivity; Mexico.

\section{Introducción}

¿Cuál es la función social de la educación superior en países como México? ¿Es verdad que promueve la movilidad social o solamente es el cemento para una mayor estratificación social? Para poder responder a estas preguntas se necesita conducir estudios empíricos para saber cómo cambian los niveles de ingreso, ocupacional y educativo de los egresados universitarios en comparación con sus padres, con el tiempo y entre ellos mismos.

pág. 8 
A esto se le llama la "movilidad social objetiva" y en México, hay un cuerpo de conocimientos bastante sólido en este sentido (véanse Cortés et al. 2007; Solís, 2012; Vélez Grajales et al. 2015; Salazar et al. 2019).

La "movilidad social subjetiva" ha sido menos analizada (véase Durán y Soloaga, 2015) y es aquí donde se circunscribe este artículo. Para emprender estudios de este tipo, Durán y Soloaga (2015) advierten que se tienen que tener en cuenta dos puntos. Por un lado, la 'brecha aspiracional'4 que indica la diferencia entre la situación actual del individuo y la situación a la que desea llegar o alcanzar, o el esfuerzo que realizarán para alcanzar sus metas fijadas por sus aspiraciones. Por el otro, están las percepciones, las cuales pueden determinar las acciones que deberán llevar a cabo los sujetos para alcanzar sus metas. La percepción, como elemento subjetivo, dicen Durán y Soloaga (2015) pueden impulsar o cohibir la acción.

De este argumento se deriva una importante observación: la combinación de variables objetivas con las de tipo subjetivo puede enriquecer el estudio de la función social de la educación, específicamente, en los patrones de movilidad social. Esto es así porque los individuos no sólo actuamos con base en los estímulos del exterior, sino también en lo que cotidianamente pensamos y construimos mentalmente.

El objetivo entonces de este artículo es indagar cómo han cambiado las percepciones de seis egresados de la Universidad Tecnológica Tula-Tepeji (UTTT) en México, a partir de sus trayectorias educativas, ocupacionales y económicas. Para dar cuenta de estos cambios a lo largo del tiempo, se diseñó un estudio de tipo longitunidal de panel para poder recabar información del mismo grupo de egresados en dos tiempos distintos (T1=2000 y T2=2019).

Se consideran como variables que describen a la movilidad subjetiva cuatro: (a) percepción del grado de libertad en distintos momentos de su trayectoria de vida, (b) percepción sobre los cambios experimentados respecto a sus características socioeconómicas de hogar de origen, (c) percepción sobre sus logros y (d) las aspiraciones sobre educarse para conseguir un empleo, las actuales y sobre lo que ahora desean para sus hijos.

Los resultados muestran que las condiciones reales de ascenso social de los egresados han modificado a su vez sus percepciones sobre la función de la educación y por esto, ellos empiezan a modificar ciertas acciones para otorgar mayor libertad a sus hijos de

\footnotetext{
${ }^{4}$ Deriva del concepto de 'ventana de aspiraciones', la cual se define como un punto de referencia para que un individuo construya sus aspiraciones.
} 
elegir sus propios caminos educativos que ellos mismos valoren y tengan razón de valorar como establecería Amartya Sen.

El texto está dividido en cuatro partes principales. En el primer apartado se repasan los principales estudios sobre movilidad social para México y se ubica a la educación dentro de ellos. En el segundo, se hace la posición teórica del artículo. Se explican cuatro razones para elegir el enfoque de las capabilities (Sen, 1985, 1987, 1999, 2009) en este estudio de movilidad subjetiva. Estas razones son de tipo conceptual, ontológico (centralidad en el individuo), con énfasis en la racionalidad y la profunda complementariedad entre agencia humana y estructura social. En la tercera sección se describe el diseño metodológico donde se especifica que es un estudio de tipo longitudinal de panel, que se utilizan dos instrumentos de recolección de datos (encuesta y entrevista en forma de historia de vida) y los problemas para entrevistar al mismo grupo en dos tiempos distintos. La cuarta parte presenta el análisis y la discusión de los resultados y se divide a su vez en dos partes. Una que da cuenta de cómo cambiaron las percepciones de los egresados universitarios a través del tiempo y la segunda es concerniente con las elecciones razonadas de estos jóvenes en referencia con sus hijos y el cambio en sus condiciones reales de desarrollo.

El texto cierra con las conclusiones principales y advierte de la necesidad de conducir estudios con poblaciones más extensas. Finalmente, sostenemos, de lo que se trata es de saber bajo qué condiciones la educación superior de corte tecnológica - que ha sido la opción principal para ampliar la cobertura en México - puede constituir o no la fuerza real detrás de la movilidad social.

\section{Movilidad social, subjetividad y educación}

¿Es verdad que la escolaridad promueve la movilidad social? Pese a los valiosos esfuerzos de múltiples autores (véanse Cortés et al. 2007; Solís, 2012; Blanco et al. 2014) por responder a esta pregunta, aún hay serias dudas sobre cómo los estudios de nivel universitario contribuyen al mejoramiento ocupacional, de ingresos y académico de una generación a otra o dentro del mismo grupo social.

México le ha apostado históricamente a la expansión educativa con el propósito de mejorar sus niveles de bienestar y desarrollo, sin embargo, también hay serias dudas sobre la función social de la educación superior: ¿es reproductora de desigualdades o motor de movilidad ascendente? Salazar et al. (2019) afirman que la "educación debería ser uno de los principales motores de la movilidad social, pues da capacidades a las personas para que tomen mejores decisiones sobre sus vidas y así, puedan alcanzar mayores niveles de 
bienestar." (Salazar et al. 2019, p. 27). No obstante, pese a la expansión de servicios educativos en México, los autores también observan que ésta es de "corto alcance".

Solo $5 \%$ de los hijos de padres sin escolaridad logran estudiar una licenciatura, en comparación con $64 \%$ de los hijos de padres con estudios universitarios. Hijos cuyos padres cuentan con esta educación, alcanzan la formación profesional a una tasa doce veces mayor que quienes provienen de padres sin escolaridad (Salazar et al. 2019, p. 28).

¿Qué factores subyacen a la inmovilidad educativa que reporta Salazar et al. (2019)? ¿Decisión racional de los padres sobre la rentabilidad educativa a futuro? ¿Aspiraciones y tradiciones familiares? ¿Falta de oportunidades reales para ascender social y académicamente? Racionalidad de los propios actores, normas sociales o "cultura", así como condiciones reales de crecimiento personal pueden explicar los datos que reportan Salazar et al. (2019).

Vélez et al. (2014), por su parte, estudian a la movilidad social incluyendo la variable de percepción, la cual, refiere a un campo subjetivo para el desarrollo de las personas. Esto es muy loable, pues se pueden empezar a pensar en cómo una actitud del propio individuo opera frente a los retos de la vida. Este tema ha preocupado a los estudios sociológicos por siglos: "lo que el individuo piensa y cómo actúan sirve para modificar las condiciones externas (contexto) donde se desenvuelve o es a la inversa" (véase Baert, 1998).

Durán y Soloaga (2015) enriquecen este campo al desarrollar un estudio sobre percepciones y la movilidad social en México. Su investigación se basa en un modelo de aspiraciones y desigualdad (de Genicot y Ray, citado por los autores), el cual asume que los individuos conocen la distribución de los ingresos entre la población y dicho conocimiento es útil en la formación de sus aspiraciones. En referencia a la percepción del entorno, Durán y Soloaga (2015) afirman que ésta puede influir en la interpretación de la distribución de los ingresos conocida. Estos autores hacen una clasificación útil para relacionar el nivel de percepción con el de las aspiraciones o expectativas. De tal manera, mencionan que puede haber "individuos pesimistas e individuos optimistas" donde los primeros tienden a acumular más años de escolaridad que los segundos. Al analizar, por otro lado, las aspiraciones educativas encuentran que cuando el padre posee un año menos de escolaridad, ésta se "compensa" con una percepción de movilidad ascendente. Asimismo, en términos de situación socioeconómica relativa percibida obtienen que ésta es menor a la real. Esta disparidad entro lo objetivo y lo subjetivo justifica poner atención en ambas dimensiones para analizar los patrones de movilidad social. Si se perciben déficits en los planos educativos, ocupaciones y monetarios, el individuo tenderá a actuar de una u otra manera que es importante comprender. 
La racionalidad de los sujetos, como lo han dicho múltiples actores desde Max Weber hasta Amartya Sen (2009), es central para comprender mejor la acción y con ello, fenómenos como el de la movilidad social y la función de la educación en el proceso de desarrollo humano. Volveremos a este punto más tarde, mientras es importante seguir analizando el papel de la subjetividad en la educación.

En este sentido, Villa-Lever (2016) estudió la movilidad subjetiva entre jóvenes universitarios de último año en tres dimensiones de percepción: (1) posición socioeconómica, (2) posición laboral y (3) posición de prestigio. En las tres, encuentra una movilidad ascendente, incluso, mientras más bajos sean los ingresos familiares y menor sea el nivel escolar de los padres, las expectativas de movilidad de los jóvenes universitarios son más altas. Esto muestra, otra vez, la importancia de estudiar conjuntamente variables objetivas (ingreso, antecedente escolar del padre) con la dimensión subjetiva (percepción) y las aspiraciones a futuro.

Como todo resultado académico, los estudios sociales que incorporan la dimensión subjetiva y objetiva son producto de críticas. Por ejemplo, Jaramillo (2016) hace una revisión entre bienestar subjetivo y objetivo para el caso de México y sostiene que existen sesgos y errores de la medición, en el uso y en la construcción del bienestar objetivo. También argumenta que se puede evaluar la percepción de movilidad social al comparar el nivel de vida entre el hogar de origen y el actual y ésta tiene un efecto positivo directo sobre el bienestar subjetivo. Más allá de validar o no el cuestionamiento de Jaramillo, lo que aquí es claro es que al combinar las variables subjetivas (percepción) con objetivas se puede comprender mejor la acción individual dentro de un marco de análisis más amplio en donde lo que interesa saber es porqué la gente "sale adelante", progresa, se desarrolla y logra propósitos y estilos de vida que valora y tiene razón para valorar, como diría Sen (1999).

La racionalidad práctica de los individuos - que en parte contiene a la percepción como variable subjetiva - es importante para comprender los distintos patrones de movilidad social. Este es uno de los argumentos que justifican la realización de este artículo.

\section{2. ¿Por qué utilizar el enfoque de capabilities en estudios de movilidad?}

Para poder estudiar la movilidad social, Westoff et al. (1960) sugieren que hay que considerar tres planos como unidades de análisis: (1) individuo, (2) familia y (3) grupo social. Luego, la dirección de la movilidad. Existe, por un lado, la movilidad "horizontal" y por otro la "vertical" La primera se refiere a la transición de un individuo dentro de un grupo social, mientras que la segunda se refiere al movimiento de una persona de un estrato social a otro. 
Otros "puntos de referencia" es la movilidad intrageneracional, por un lado, y por otro, la intergeneracional. Esta última indica el ascenso o descenso de una persona con respecto a lo que lograron sus padres, mientras la primera refiere al movimiento que ha tenido una persona desde su primera ocupación laboral hasta la actual.

Westoff et al. (1960) también hacen énfasis en dos variables para conducir los estudios sobre movilidad social. En primer lugar, la cantidad de personas que han tenido movilidad y en segundo, la percepción. Frente a medidas "objetivas" como el ingreso o el tipo de ocupación, es importante poner atención en lo que los individuos piensan, sienten o esperan, ya que hay, según Palomar y Lanzagorta (2005) variables psicológicas y sociales capaces de predecir la movilidad social.

No hay seguridad si "predecir" es un buen término, sin embargo, es claro que lo subjetivo ("psicológico") cuenta para el análisis social. Por ello, es importante mencionar el trabajo de Van den Berg (2011) quien manifiesta que la definición de movilidad social, así como las maneras tradicionales de estudiarla, son rígidas y no tan atinadas. Siguiendo el trabajo de Pierre Bourdieu, Van den Berg (2011) observa con razón que no existe una estructura de clases determinada solamente por la educación, el ingreso o la ocupación, sino que existe un capital simbólico que amplía la perspectiva hacia una definición de movilidad o estructura de clases menos estrecha.

Van den Berg (2011) encuentra, en su estudio de corte cualitativo, que sus informantes (mujeres marroquíes migrantes) determinaron con base en su propia historia de vida si han "mejorado su clase" o "han escalado la escalera social". Los resultados, según este autor, apuntan a que las mujeres modificaron sus aspiraciones después de migrar. Pasaron de tener como plan de vida ser esposas y amas de casa a querer trabajar e ir a la escuela para conseguir mejores empleos. El cambio de condiciones de vida reales (objetivas) cambió su manera de pensar. ¿Se enfocarán estas mujeres hacia la realización de nuevos estilos de vida que valoran - y tienen razón de valorar, como diría Sen $(1999,2009)$ ¿Habrá oportunidades reales para ellas? Esto sería materia de otro análisis, pero no obsta decir que la movilidad social está inserta en una línea de tiempo donde los individuos eligen de maneras distintas y actúan por variadas razones que se deben explorar y comprender más ampliamente.

La actuación del ser humano hace al enfoque de capabilities, desarrollado por Amartya Sen (1985, 1987, 1999, 2009), Martha Nussbaum (2000) y otros más, resulta pertinente para estudiar la movilidad social, pues se centra en el individuo como la unidad de preocupación moral, un "individualismo ético", en palabras de Robeyns (2014), ni metodológico ni mucho menos ontológico. 
Según Durán y Soloaga (2015), cuando los individuos analizan sus posibilidades para tomar decisiones sobre ellos mismos o sobre sus hijos, lo hacen bajo la percepción de su entorno y eso afecta su brecha aspiracional. Esta brecha representa la distancia entre su estatus actual y el punto al que desean llegar. Asimismo, el esfuerzo que los individuos decidan aplicar para el logro de sus metas o aspiración específica, será con base en la decisión que tomaron según su percepción (itálicas de los autores). La percepción (lo subjetivo) surge del individuo, no del grupo o la colectividad.

Lopreato y Hazelrigg (1970), por su parte, sostienen que la movilidad social vertical implica un cambio en las actitudes sociopolíticas o de resocialización ${ }^{5}$ de los individuos. Es decir, a medida que cambiamos de situación social (clase, grupo o estatus), adquirimos una capacidad de elegir (preferir) distinta y esto refiere a campos de actuación o habitus - en términos de Bourdieu (1997). Si esto es verdad, aquí estaría demostrada esa "profunda complementariedad" entre agencia individual y estructura social de la que también habla Sen (1999) en su obra Development as freedom o el sociólogo británico Anthony Giddens.

La teoría de la estructuración de Giddens, según Baert (1998, p. 100), trata de trascender la división clásica entre lo "micro y lo macro" y para ello considera a las personas como seres conocientes (knowledgeable) y enfocados a propósitos (purposive). Si se suscribe a esta posición ontológica, lo subjetivo tiene un lugar central a considerar en la explicación social ${ }^{6}$.

Kelly y Kelly (2009) confirman este punto al observar que la "movilidad subjetiva" afecta de manera importante las actitudes y percepciones de los individuos, aún controlando variables de educación, ingreso, ocupación y crecimiento económico. Estos autores, además, señalan que la movilidad subjetiva es tan importante que una de sus consecuencias es que se incremente la tolerancia a la desigualdad. Es decir, si uno percibo que está progresando socialmente cuando no es verdad, no actuará para revertir tal condición de injusticia estructural.

Dado que interesa centrarnos en lo subjetivo, una perspectiva individualista es necesaria. Esta es la primera razón para elegir como marco de análisis el enfoque de las capabilities. La segunda razón, tiene que ver con la complementariedad que observa Sen entre agencia individual y estructura social. $Y$ hay una tercera más que tiene que ver con el aporte conceptual de Sen (1985).

\footnotetext{
${ }^{5}$ Cambio de las preferencias que inicialmente fueron forjadas dentro de una cierta estructura social. ${ }^{6}$ Amartya Sen, junto con Eric Maskin y Barry Mazur, han impartido el curso sobre Objetividad y Subjetividad en la Universadad de Harvard.
} 
Para este autor, ni los ingresos económicos ni la felicidad o el placer (utilidad) pueden ser una métrica adecuada para realizar valoraciones normativas (justicia, desarrollo, igualdad). La salida a esta limitación es la capability que puede ser definida como "las distintas combinaciones de funcionamientos (functionings) que es posible alcanzar (1999, p. 83). Los functionings forman la capability y pueden ser estados de existencia (estar o no nutrido) y actividades (participar en la vida de la comunidad) $(1985,1999)$. La capability equivale, según Sen, a la "libertad sustantiva" que a su vez refiere a la habilidad real de la persona para hacer diferentes cosas que valora. El enfoque de las capabilities, se centra en las "vidas humanas y no sólo en los recursos de la gente" (2009: p.253). El concepto de capability o libertad será utilizado en el análisis empírico cuyos informantes son seis egresados de una universidad tecnológica de México.

El énfasis en la racionalidad (rationality) de las personas para elegir lo que "valoran y tienen razón de valorar" (Sen, 1999, p. 293), es la cuarta razón para aplicar el enfoque basado en la libertad. Lo subjetivo es expresado meramente por el individuo y le ofrece a éste la oportunidad de elegir de maneras diferenciadas dentro de su propio contexto.

A continuación, se explica cómo se va a operacionalizar el enfoque de las capabilities, una vez que se han identificado sus ventajas teóricas.

\section{Diseño metodológico}

Este artículo responde a la pregunta de cómo han cambiado las percepciones (subjetividad) y aspiraciones de seis jóvenes egresados de una universidad tecnológica ubicada en México, a partir de que se involucraron en un proceso educativo y laboral. Más específicamente, ¿qué libertades (capabilities) desarrollaron?, ¿cómo ha cambiado su manera de pensar a partir de que cumplieron sus propósitos?, y ¿qué planean a futuro?

Junto a los rigurosos trabajos que estiman la probabilidad de moverse social y ascendentemente con muestras probabilísticas o poblaciones extensas, Duru-Bellat y Kieffer (2008) señalan que:

[...] el enfoque para estudiar la movilidad subjetiva podría no estar limitado a un estudio de datos estadísticos, a pesar de la robustez que represente; se tienen que tomar en cuenta las interpretaciones de los individuos sobre su situación [...] al estudiar la movilidad subjetiva, es relevante tomar en cuenta todos los tipos de trayectoria, aquéllas realizadas y aquéllas planeadas y proyectadas, manteniendo el tiempo como un continuo en lugar de romperlo. (Duru-Bellat y Kieffer, 2008, p. e3). 
Para capturar las "interpretaciones de los individuos" y estudiar la "movilidad subjetiva" nos concentraremos, precisamente como recomiendan Duru-Bellat y Kieffer (2008), en las trayectorias "realizadas" y "proyectadas" de seis jóvenes egresados de la Universidad Tecnológica de Tula Tepeji (UTTT), Méxicoº

¿Por qué la UTTT?, Se eligió esta universidad bajo dos criterios. Primero, representa un estudio de caso. Según Bryman (2001), el estudio de caso permite analizar las condiciones contextuales y reales que rodean el objeto de estudio, en este caso: la movilidad social subjetiva en seis egresados universitarios. Segundo criterio: en el año 2000, se condujo una investigación para saber si la educación superior ampliaba las capabilities de los egresados de la UTT (ver Flores-Crespo, 2005). Por tanto, se tenía ya construida una base de información sobre educación y libertad que podría aprovecharse en un segundo momento. Esto implicó que se diseñara un análisis longitudinal de panel con el objetivo de indagar cómo habían cambiado los patrones de desarrollo en el tiempo, estableciéndose el Tiempo 1 en 2000 y el Tiempo 2 en 2019. Los estudios longitudinales, según Hernández Sampieri et al. (2008), permiten realizar inferencias acerca del cambio y, en este caso particular, podrían indicar algún patrón de movilidad social ya que se estudiaba al mismo grupo de egresados, pero en dos momentos distintos.

En el Tiempo 1 (2000) se pudo encuestar de manera aleatoria a 177 egresados y aunque se trató de localizar a la mayoría de éstos en el Tiempo 2 (2019), localizarlos fue particularmente difícil. Sólo se logró tener respuesta de seis jóvenes, lo que hizo variar el tipo de estudio, las técnicas de recolección de datos y de análisis.

Debido al reducido número de casos, se optó por recoger datos tanto con el cuestionario originalmente aplicado en el Tiempo 1 (2000) como a través de una entrevista semi estructurada en su variante de historia de vida. Janesick (2013) afirma que la historia de vida permite identificar los puntos de inflexión existentes en la trayectoria de vida de las personas y, con ello, se consigue información valiosa sobre el cambio en sus percepciones y aspiraciones.

Para poder capturar los cambios a través del tiempo, el cuestionario también registró cambios para poder recabar información retrospectiva y actual e incorporar preguntas sobre la percepción del cambio en condiciones socioeconómicas y la libertad (capability) real y percibida en distintos momentos. El cuestionario se aplicó para el Tiempo 2 (2019) entre mayo

\footnotetext{
7 Para conocer a fondo los antecedentes, principios, logros y limitaciones del modelo de las universidades tecnológicas mexicanas, véase Flores-Crespo, 2009 o Flores-Crespo y Mendoza, 2013.
}

pág. 16 
y junio, y en julio y agosto se realizaron las entrevistas. Finalmente, la muestra no probabilística de informantes quedó como lo expone la Tabla 1.

Tabla 1. Perfil de los informantes ${ }^{8}$

\begin{tabular}{|c|c|c|c|c|c|c|c|}
\hline Código & $\begin{array}{l}\text { Edad } \\
\text { actual }\end{array}$ & $\begin{array}{c}\text { Nivel } \\
\text { educativo } \\
\text { alcanzado }\end{array}$ & $\begin{array}{c}\text { Ocupación } \\
\text { actual } \\
\text { (T1=2019) }\end{array}$ & $\begin{array}{l}\text { Primer } \\
\text { empleo }\end{array}$ & $\begin{array}{c}\text { Ramo de } \\
\text { la empresa }\end{array}$ & Título TSU & $\begin{array}{c}\text { Año } \\
\text { de } \\
\text { egreso }\end{array}$ \\
\hline M1 & 40 & $\begin{array}{l}\text { Estudiante de } \\
\text { doctorado en } \\
\text { Ingeniería de } \\
\text { Sistemas } \\
\text { Empresariales }\end{array}$ & $\begin{array}{l}\text { Profesor } \\
\text { tiempo } \\
\text { completo }\end{array}$ & Recepcionista & Universidad & $\begin{array}{l}\text { Procesos de } \\
\text { producción }\end{array}$ & 1999 \\
\hline M2 & 39 & $\begin{array}{l}\text { Licenciatura } \\
\text { en } \\
\text { Contabilidad }\end{array}$ & $\begin{array}{c}\text { Jefa del } \\
\text { Departamento } \\
\text { de Calidad }\end{array}$ & $\begin{array}{c}\text { Asistente } \\
\text { Administrativo }\end{array}$ & Universidad & $\begin{array}{l}\text { Contabilidad } \\
\text { Corporativa }\end{array}$ & 1999 \\
\hline M3 & 44 & TSU & $\begin{array}{l}\text { Ama de casa } \\
\text { y ventas por } \\
\text { catálogo }\end{array}$ & $\begin{array}{l}\text { Técnico } \\
\text { calificado }\end{array}$ & $\begin{array}{c}\text { Negocio } \\
\text { propio }\end{array}$ & $\begin{array}{c}\text { Mantenimiento } \\
\text { industrial }\end{array}$ & 1995 \\
\hline H1 & 45 & TSU & $\begin{array}{l}\text { Supervisor de } \\
\text { mantenimiento }\end{array}$ & $\begin{array}{l}\text { Técnico } \\
\text { calificado }\end{array}$ & Alimentaria & $\begin{array}{c}\text { Mantenimiento } \\
\text { industrial }\end{array}$ & 1995 \\
\hline $\mathrm{H} 2$ & 44 & $\begin{array}{l}\text { Ingeniería } \\
\text { Mecánica }\end{array}$ & $\begin{array}{c}\text { Jefe de } \\
\text { operación }\end{array}$ & $\begin{array}{c}\text { Obrero } \\
\text { calificado }\end{array}$ & Química & $\begin{array}{c}\text { Mantenimiento } \\
\text { industrial }\end{array}$ & 1995 \\
\hline H3 & 39 & $\begin{array}{l}\text { Ingeniería en } \\
\text { Tecnologías } \\
\text { de }\end{array}$ & $\begin{array}{c}\text { Área de } \\
\text { Transmisión } \\
\text { Eléctrica }\end{array}$ & $\begin{array}{l}\text { Técnico } \\
\text { calificado }\end{array}$ & Electricidad & $\begin{array}{l}\text { Procesos de } \\
\text { producción }\end{array}$ & 1999 \\
\hline
\end{tabular}

Fuente: Elaboración propia.

\section{Análisis y discusión de resultados}

Esta sección relaciona lo expuesto en los apartados teóricos (1 y 2) con la base empírica. La pregunta guía es: cómo la educación podría compensar las influencias negativas de los orígenes sociales para dar cuenta de un proceso de movilidad social ascendente, ¿o a caso la formación universitaria es inocua e irrelevante?

${ }^{8} \mathrm{~A}$ todos ellos se les prometió guardar anónimo los nombres de su empresa y el suyo propio, de ahí que se les haya asignado una clave en donde $\mathrm{M}$ refiere al sexo femenino y $\mathrm{H}$ al masculino. 
Es importante mencionar que el modelo de universidades tecnológicas de México fue creado en 1991 con el objetivo de captar jóvenes de los estratos sociales más desfavorecidos, cuyas oportunidades de estudiar eran bajas. Este modelo ofreció, en sus inicios una educación de tipo vocacional y de corta duración (dos años) pudiendo los jóvenes egresar con el título de Técnico Superior Universitario (TSU).

En 2006, el ex subsecretario de Educación Superior de México, Julio Rubio, afirmó que "nueve de cada diez jóvenes fueran los primeros en sus familias en tener la oportunidad de realizar estudios superiores" (Rubio, 2006, p. 165). Es decir, se registró una movilidad educativa intergeneracional.

No se puede negar que la movilidad educativa intergeneracional representa un avance significativo, sin embargo, falta por indagar si los estudios en la UTTT les permitieron a los egresados ampliar sus libertades (capabilities). De esto da cuenta el trabajo de FloresCrespo (2005) para el año 2000, pero ¿qué pasó después? ¿Cómo cambiaron sus ocupaciones, ingresos y nivel educativo a través del tiempo? Flores-Crespo y Rodríguez Arias (en prensa) muestran que estos seis egresados lograron movilidad social objetiva.

Según estos autores, las condiciones socioeconómicas de los seis egresados mejoraron a través del tiempo, tal como muestra la Gráfica 1. El ingreso mensual en el primer empleo fue más bajo que el registrado en el Tiempo 1 (2000) y más alto en el Tiempo 2 (2019) (Flores-Crespo y Rodríguez Arias, en prensa).

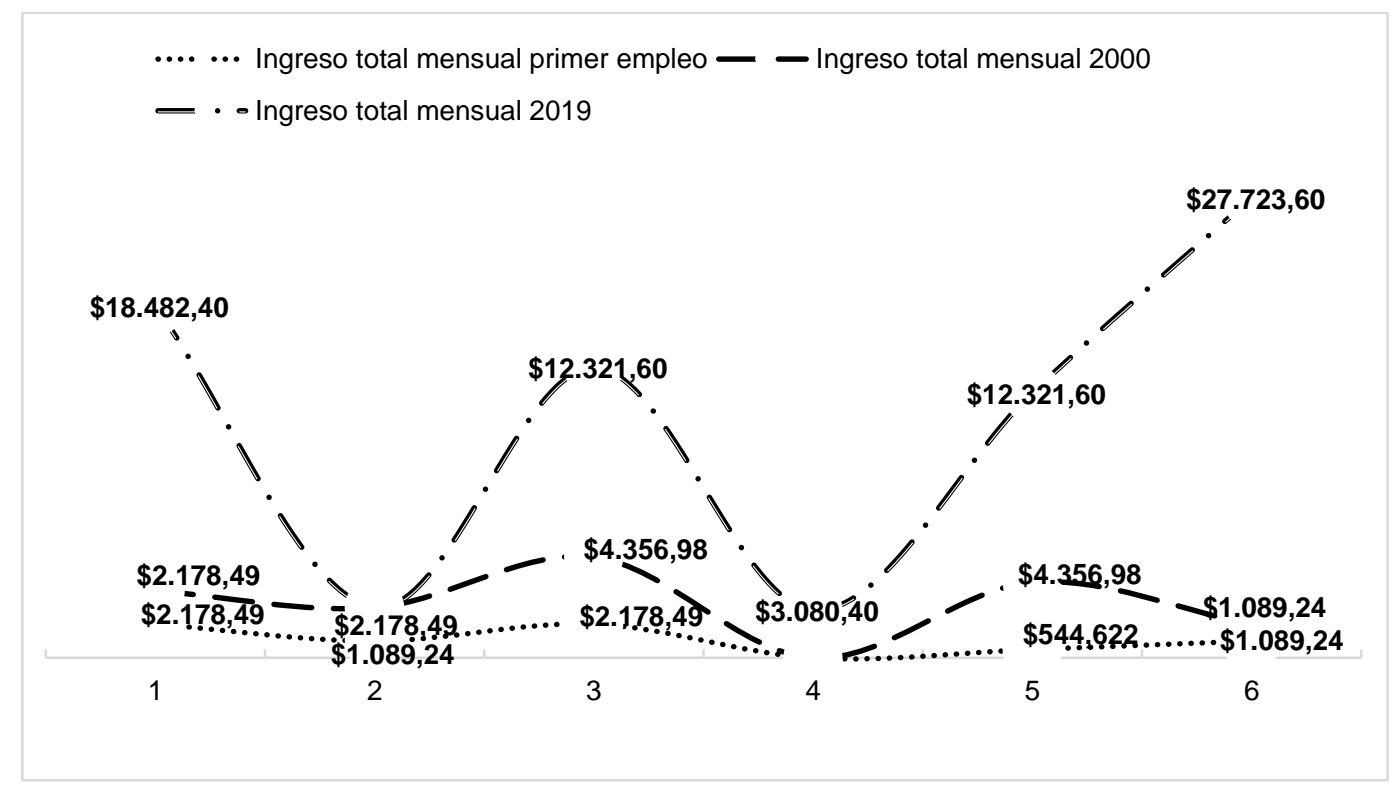

Gráfica 1. Cambios en el poder adquisitivo de los egresados a través del tiempo.

pág. 18 
Fuente: Elaboración propia. Pesos constantes (MXN), base julio 2018. Nota: No existen los tres datos para las seis observaciones.

Asimismo, con respecto a la movilidad educativa y ocupaciones, Flores-Crespo y Rodríguez (en prensa) también muestra que el total de egresados en su estudio $(n=6)$ mostraron movilidad educativa respecto a la madre. En cuanto al padre, solamente una egresada no superó el nivel educativo del padre (licenciatura) y sus ocupaciones eran mejores que aquellos que tuvieron sus padres. Esto muestra que la escolaridad no fue inocua no irrelevante para estos seis jóvenes. Habrá ahora que indagar a poblaciones más extensas para constatar o rechazar lo observador por Flores-Crespo y Rodríguez (por aparecer).

Pero indaguemos más allá de las variables objetivas de ingreso, nivel escolar y ocupación. La siguiente sección hace alusión al tema de la subjetividad.

\section{A. Percepciones de libertad a través del tiempo}

Para explorar el componente subjetivo en los egresados de la UTTT, se les preguntó de manera retrospectiva cuándo se sentían más "libres" de hacer las cosas que les gustaban (valoraban, en términos de Sen, 1999). Marcamos tres tiempos en la entrevista: (1) como estudiante, (2) al egresar y trabajar, y (3) ahora (T2). Un joven que había estudiado Mantenimiento Industrial y ahora es ingeniero, respondió así:

¡Wow! Qué pregunta tan interesante. No, pues cuando era estudiante definitivamente tenía más libertad de hacer muchas, muchas cosas $(\mathrm{H} 2$ itálicas nuestras)... por ejemplo, mi primer trabajo [...] el Jefe de Mantenimiento, aparte de que me daba mucha libertad [...], no me limitaba, por ejemplo de que "no te metas a ese departamento" o "no hagas esto", no, es "haz lo que consideres que es para que podamos estar bien" y esa es la libertad que, que tuve en aquéllos entonces, quizá por eso aprendí mucho de todo, o sea desde la cuestión administrativa, el mantenimiento mecánico, eléctrico.

Similar a este egresado, otra joven, que no pudo entrar a estudiar en el Colegio Militar pero que tuvo una segunda oportunidad en la UTTT, expresó:

Pues prácticamente cuando estudié. Tenía la libertad total de hacer lo que yo quisiera. Cuando egresé no tanto, porque en el momento en que egresé, nada más fueron escasos tres meses y me incorpor[é] al campo laboral... En el campo laboral, todavía tuve un poquito de libertad de hacer las cosas, porque todavía no me casaba y todavía no tenía a mi hija.

Cuando [tuve] a mi hija, pues me [cohibí] un poquito el hacer varias cosas, aunque tuve el apoyo de mi mamá, pero aún así hay una responsabilidad ya ahí marcada $[\ldots]$ 
Actualmente, pues estoy dedicada al cien por ciento a mi hija y ahorita sí siento que es como que una barrera para poder hacer lo que uno quiere realizar. Pero el tiempo más idóneo en donde pude hacer todo lo que quería, era cuando estudiaba (M2 itálicas agregadas).

Para las mujeres egresadas, desempeñar el papel de mamá está en constante interacción con otros roles sociales que deben cumplir. Otra egresada cuyo primer puesto fue de recepcionista y ahora es profesora de tiempo completo en la UTTT respondió así a nuestra pregunta original:

Pues creo cuando egresé me sentía más libre para hacer más cosas que me [gustaban]

Investigador: ¿Por qué?

Egresada: Porque no estaba casada; porque [...] me fui a vivir fuera [de casa] de mis papás... (M1)

Y ahora que, pues, ya estoy casada, que tengo hijos, que estoy estudiando, que trabajo, pues mis responsabilidades están súper elevadas y pues, tengo que sacrificar algo que me encanta [que] es hacer ejercicio. Ya no lo puedo hacer como lo hacía antes, y entonces pues por eso no me siento tan libre como antes que hacía ejercicio, que me iba mucho de viaje, paseaba bastante, pues me iba a bailar, dormía lo que quería... (M1 itálicas agregadas).

Otro egresado de 45 años, y con un puesto de supervisor de mantenimiento, reflexiona detenidamente ante la pregunta y agrega:

¿Más libre? Pues en el momento en el que empecé a trabajar, pero sobretodo más en estos tiempos de crecimiento personal, de crecimiento de conocimientos. No solo ha sido [conocimiento] técnico, sino también social. Empiezas a ver métodos de cómo tratar a la gente, cómo manejarla, cómo conocerla, que es un factor importante cuando lideras un grupo de trabajo $(\mathrm{H} 1)$.

Ante la pregunta retrospectiva que se les hizo hasta aquí los jóvenes parecen tener una percepción variada de la libertad de acuerdo con las cosas que pudieron hacer e, interesantemente, de acuerdo con las responsabilidades que tenían que cumplir (cuidar hijos, por ejemplo). Esto varió claramente con el tiempo. A mayor tiempo, mayores responsabilidades y con eso una percepción restringida de la libertad.

Otra joven que manejaba su propio negocio, y que fue la única de la muestra que no cursó un nivel escolar más allá del TSU, percibe que ahora tiene más libertad para hacer lo que ella valora. ¿Por qué? A contnuación su respuesta 
[Ahora] ya no tengo la presión del tiempo, de que tenía que terminar rápido la carrera y empezar a generar ingresos. $Y$ ahora porque estoy en un lugar que me brinda todas las oportunidades de trabajo [...] He ido a hacer entrevistas laborales para entrar a otro trabajo, pero cuando veo los sueldos, digo: "no". Como nada más soy TSU [digo] no, no me conviene y [además] veo el horario y no (M3).

De este testimonio, vale la pena advertir la "racionalidad razonada" del individuo. No desea trabajar donde paguen poco y labore tiempos largos. Por eso, valora continuar con su negocio y parece dueña de su tiempo.

Una racionalidad distinta fue expresada por otro egresado que claramente respondió: "me siento a lo mejor más libre ahorita, porque a lo mejor la vida es un poco más estable" (H3 itálicas agregadas).

Además de la libertad (capability) que han percibido en diferentes momentos para hacer lo que valoran, se puede analizar también lo que los egresados perciben sobre sus logros o éxito alcanzados. Para personas como el ingeniero en tecnologías de manufactura (H3), alcanzar estabilidad económica representa una sensación de bienestar más de acuerdo a lo que define Pigou (1932), pero para otros es más una cuestión de tener un balance, no necesariamente en términos económicos. Así lo comenta una mujer egresada de la UTTT que actualmente cursa un doctorado:

Pensaba que estudiar más me iba a dar un rango más de puesto. Hoy en día he descubierto que eso no te lo da [...] más bien lo siento como satisfacción propia y pues como ejemplo a seguir para mis hijos, motivarlos y luchar por darles también un ejemplo a los alumnos, de que podemos ser mejor y que podemos pensar diferente (M1 itálicas agregadas).

Aquí hay dos cuestiones interesantes. Por un lado, percibe que la educación no le dará un puesto más alto a futuro y por otro, asume que la educación es para poder pensar diferente. Es decir, la educación moldea la racionalidad de las personas. Esta destacada egresada va a fondo con su testimonio al preguntarle por sus condiciones de vida.

Mis condiciones económicas creo que son las necesarias y que tengo un bienestar económico saludable (sic). Siento que he trabajado mucho, siento que he logrado muchas cosas [...], pero la verdad es que yo creo que estoy muy bien, porque me siento con calidad de vida [...] estoy cerca de donde vivo, estoy cerca de poder ver a mis hijos, estoy en un lugar donde me gusta trabajar y que me gusta lo que hago y entonces, el tener ese equilibrio o ese bienestar compensa el que yo pudiera ganar más en otro rango o en otro (M1, itálicas agregadas). 
Este testimonio constata lo dicho en los apartados teóricos: la combinación de variables objetivas (ingreso) con las subjetivas (percepción sobre la "calidad de vida") no debe de omitirse en los estudios sobre movilidad social. Esta joven prefiere tener "equilibrio" en su vida que tener un ingreso más alto. ¿Cómo captura esta elección un modelo clásico de movilidad social ascendente? Tal "racionalidad razonada" del individuo - en este caso una mujer - debe considerarse en futuros estudios sobre movilidad social.

Con respecto a las expectativas y aspiraciones de los egresados, otro de los consultados aseguró que se han cumplido, pese a que sus capabilities fueron limitadas. Él ilustra el punto así:

Creo que si he cumplido con mi perspectiva de vida que en aquel momento o en aquel tiempo pensaba yo, tal vez no fue la carrera que yo hubiera querido, pero la que tengo me dio y me está dando buenas satisfacciones, tanto económicas como personales, entonces, creo que estoy en un nivel de vida bueno ( $\mathrm{H} 1$ itálicas agregadas).

Este testimonio vuelve mostrar que la educación superior ofrecida por la UTTT no fue irrelevante, a pesar de que no quería estudiar ahí. En otras palabras, aunque en un primer momento no tuvo la capacidad o libertad de elegir su universidad, ha cumplido con su "perspectiva de vida" y posee, según su percepción -y los datos "objetivos" mostrados en Flores-Crespo y Rodríguez (en prensa)- un mejor nivel de vida que el de sus padres y del que percibía cuando inició su carrera profesional.

En el inicio de su vida profesional, el título de TSU -dijo- lo limitó. En palabras de este joven:

sí hubo unas oportunidades que pudieron mejorar eso [nivel de vida], que no se pudieron dar precisamente por la carrera que yo tenía ¿no? Había puestos ya a un nivel... no tanto gerencial pero si un poco más abajo, que no pude yo tomar por la carrera que yo tenía, ese fue uno de los límites $(\mathrm{H} 1)$.

A qué grado limita el título de TSU a los egresados de moverse más rápido laboralmente es un tema que debe explorarse a futuro. Desde el año 2000, han habido indicios de que una manera de "romper" esta limitante es estudiar un nivel educativo mayor (licenciatura e ingenierías) (véase Flores-Crespo, 2005).

\section{B. Elección escolar razonada: Cambios a lo largo del tiempo}


Para saber cómo se ha modificado la percepción de los egresados respecto a sus opciones escolares, se les preguntó si ahora recomendarían a sus hijos estudiar en la UTTT. Una egresada, con nivel de licenciatura, dijo que sí se lo recomendaría, de hecho, se lo había "sugerido" a su hija pero:

lamentablemente aquí [en la UTTT] no tenemos [el programa de] Criminalística Forense, y no la quito [a mi hija] de que no estudie esa carrera. De hecho, yo le decía que viniera a estudiar, tenemos Administración de Capital Humano, porque ella es muy sociable, se le da mucho esa parte" (M2).

Con relación a la oferta de carreras, otro egresado, que terminó sus estudios hace 25 años, también comentó que no le recomendaba a su hijo estudiar en la UTTT. He aquí su respuesta en extenso.

Investigador: ¿Le sugeriste a tu hijo que estudiara en la UTEQ o no?

Egresado: No $(\mathrm{H} 1)$

Investigador: ¿No?

Egresado: No, de hecho, una de sus opciones para él era la Universidad Tecnológica.

Investigador: ¿La UTEQ?

Egresado: La UTEQ de aquí de Querétaro. Y pues mi respuesta, ahí si fue tajante mi respuesta: un no rotundo. No porque la escuela sea mala, sino porque $[. .$.$] como padre te vas dando cuenta hacia donde van dirigidos, ¿no?$

Y la UTEQ no tiene una carrera similar como Ingeniería en Sistemas o Ingeniería en Animación, entonces, no [...] Entonces dejarlo escoger esa opción [era] como, a lo mejor se oye muy feo ¿no?, pero dejarlo que se hunda un poco.

Porque como te digo, mi filosofía es hacer lo que te gusta y si te van a pagar por eso, pues está mejor ¿no? Entonces, si ahí no va a encontrar una carrera que cumpla con sus expectativas pues no, no es conveniente que estudie ahí, si su perspectiva fuera otro tipo de carrera pues tal vez si sería diferente ¿no? $(\mathrm{H} 1)$.

Ante la pregunta de si él o el hijo habían elegido la otra institución distinta a la UTTT para cursar Animación, esto respondió:

No, él [el hijo] la eligió. Él eligió la carrera, él eligió la escuela, él estuvo viendo todo eso.

Lo que creo que sí aporté - yo un poco o creo yo que aporté - es en no darles una educación, este... federal, en escuelas públicas ¿no? Creo que también eso habla del salario que yo tenía. A los dos les pude dar escuela particular hasta la preparatoria. Bueno a mi hija ahorita la secundaria y creo que también fue parte fundamental [que haya aprendido] inglés $(\mathrm{H} 1)$. 
Interesante, el padre no eligió la universidad distinta a la UTTT a la que iría su hijo. Segundo, el egresado de la UTTT posee la idea de que la escuela "federal" (pública) no satisface ciertos requisitos de calidad (inglés) y tercero, el hijo eligió una carrera con un perfil no tradicional (Ingeniería en Animación). Algo parece que ha cambiado en el tiempo.

En los estudios de movilidad social descritos en los primeros apartados, Lopreato y Hazelrigg (1970) y Van den Berg (2011) observan que las condiciones reales de vida pueden ir modificando las percepciones y aspiraciones de las personas. La movilidad social está puesta en el espacio y en el tiempo. La recopilación de datos con los egresados de la UTTT de México permite constatar esta evolución.

Los jóvenes egresados de la UTTT, que en algún momento no pudieron elegir su universidad, pero que gracias a la universidad cambiaron de niveles de ingreso, educativo y laboral, empiezan a modificar sus patrones de consumo. Ahora desean que sus hijos vayan a la escuela de sostenimiento particular o privado. El mismo egresado arriba citado $(\mathrm{H} 1)$ ahondó más sobre el valor que él le atribuye a la educación privada:

Es una ventaja el nivel económico, [pues] te permite no llevarlos a una escuela pública. Vi y comparé las diferencias porque en la escuela pública a la que yo fui no es la misma que actualmente hay. De hecho, los dos estuvieron en escuela pública, pero también antes de eso estuvieron en una escuela particular y hay mucha diferencia. Entonces bueno, las posibilidades nos permitieron que estuvieran en una escuela particular ( $\mathrm{H} 1$ itálicas agregadas).

Sin establecer claramente cuáles eran esas "diferencias" entre la escuela de sostenimiento público y una de tipo privado, es importante hacer notar que a medida que los egresados empiezan a alcanzar cierto nivel salarial, modifican su percepción de los beneficios que puede acarrear la educación. Pero la elección de escuela tiene más matices de los que uno podría pensar. Otra brillante egresada narró lo siguiente:

En un principio [mis hijos] estuvieron en una escuela privada, pero posteriormente, los pasé a una pública derivado a que yo dije, "yo me críe en pública" y dije "quiero que conozcan ambas cosas", porque independientemente de cómo sea el trato y demás, pues siento que hay cosas que sí se diferencian entre una pública y una privada (M1).

Este testimonio muestra el cambio de preferencias y decisiones a través del tiempo que los distintos individuos van haciendo. En términos de Sen, esta egresada tuvo la libertad de elegir entre enviar -o no- a sus hijos a una escuela privada del nivel básico, cosa que ella no experimentó. 
Pero no sólo los egresados, ahora profesionales, empiezan a tener un grado de libertad mayor para enviar a sus hijos al tipo de escuela que desean, también sus hijos están marcando la pauta. Un egresado cuyo primer empleo fue el de obrero y que no pudo dejar su ciudad por no desproteger a sus padres, afirma lo siguiente al preguntarle cómo visualizaba el futuro para sus hijos.

El "mayorcito" quiere terminar su carrera. Quiere seguir estudiando, él no quiere vivir acá. Dice que saliendo de la escuela va a ver a dónde se va, porque no le gusta el entorno de todo lo que representa, la inseguridad, la falta de trabajos, todo ese rollo, no le gusta y él quiere irse a trabajar... $(\mathrm{H} 2$ itálicas agregadas).

La percepción sobre el entorno está entonces influyendo una posible acción de los hijos de los egresados de la UTTT: emigrar. Interesantemente, todos los egresados encuestados para este estudio mencionan que desean que sus hijos estudien y afirman que los apoyan para que así sea. Parece que advierten un impacto real de la escolaridad en sus vidas. Incluso, algunos egresados hablaron de estar dispuestos a invertir más en ampliar el "capital cultural" de sus vástagos. Perciben que, además de la educación formal, deben realizar actividades extraescolares como una forma de potenciar su desarrollo:

[...] ahora el enfoque que yo le doy a mi hija es que sea una profesional totalmente independiente, y que ella crezca lo más que pueda [...]. Ella la he puesto a dedicarse a estudios y actividades extraculturales [...] (M2).

Invertir en actividades extracurriculares es congruente con lo observado por Plewis y Bartley (2014) en los estudios de movilidad social. Estos autores señalan que no sólo la escolaridad de los padres afecta la movilidad social de los hijos, sino la inversión en capital cultural en clases extraescolares, talleres, viajes, ya que puede cambiar la brecha entre clases sociales y grupos. Además, la influencia de los padres ${ }^{9}$ sobre las trayectorias de sus hijos es otra variable clave en el análisis de la movilidad social y constata lo que han observado otros estudios (Goldthorpe, 1996; Hansen, 2008; Stocké, 2007).

Aparte de invertir en actividades extraescolares, ¿qué más están haciendo los egresados de la UTTT para apoyar a sus hijos para mejorar sus patrones de movilidad social? Una egresada que tenía su propio negocio resaltó la necesidad de desarrollar "pasión" en lo que decidan hacer sus hijos y aclaró:

\footnotetext{
${ }^{9}$ Goldthorpe (1996) describe que cuando se pertenece a una familia con mayor nivel económico, el individuo cuenta con ciertas ventajas estructurales para optar por un título universitario, en comparación a una familia de clase media-obrera, que probablemente elija una opción vocacional. Este tipo de educación está basada en actividades manuales o prácticas.
} 
Primero que terminen una carrera en lo que ellos les gusta, que les apasiona antes de pensar en una comodidad económica, pero que les apasione (M3 itálicas agregadas)

Otros dos egresados coinciden con el argumento anterior: ofrecer a sus hijos la oportunidad de estudiar lo que a ellos mismos valoren ("les guste") y tengan razón de valorar, diría Amartya Sen (1999). En este sentido dos egresados asentaron que las cosas han cambiado entre ellos y sus hijos.

Pues igual tienen más opciones de desarrollo, de crecimiento de conocimiento. Ahora creo que sí es diferente y yo tengo [...] la carta abierta a que ellos estudien lo que realmente les guste ( $\mathrm{H} 1$ itálicas agregadas)

Pues que estudien, que sigan con su estudio, que sigan preparándose y conforme va pasando este tiempo, pues lo que a ellos les guste, apoyarlos al $100 \%$ pero en lo que ellos les guste (H3 itálicas agregadas).

También es un común denominador entre los egresados de la UTTT expresar que sus hijos deben tener la libertad de elegir sus propios caminos, y así lo ilustra el siguiente testimonio.

Pues ahora si que ya como padre pues no me resta más que apoyarlo en la decisión que tome, siempre y cuando sea buena, apoyarlo. Mi hija ahorita está en segundo de secundaria, [...], ella quiere estudiar medicina, [...] va muy muy bien en la escuela, alumno de excelencia y como padre, pues se siente uno muy orgulloso, entonces como le digo yo "lo que tú quieras y mientras en mis manos esté, yo te voy a apoyar, para que vueles lo más alto que tú quieras" [...] (H2 itálicas agregadas).

Hace algunos años esto no fue así para alguno. Ante la pregunta de porqué a una joven no le había gustado la carrera que cursó en la UTT, así respondió:

La estudié porque como no quedé en el área de Psicología de la UAQ, mis papás pensaron que hasta ahí me iba a quedar. O sea, no estábamos acostumbrados como ahora de que date un "break", que después al siguiente [año] entras [a estudiar a la universidad], sino que entras porque entras. $A$ lo que sea pero entras. Entonces lo más cercano era la UT, que no pide tantos requisitos para admisión. Entonces, mi mamá me fue a inscribir. Ella no supo ni qué carreras había, simplemente llegó y me inscribió, y al otro día me dijo "te presentas tal día, ya está tu escuela y vas" (M3).

Las cosas han cambiado con el tiempo. Una de ellas fue la posición de los padres hacia la educación de sus hijos, y la capacidad de éstos para elegir sus caminos. ¿Hubieran cambiado de parecer los jóvenes - ahora profesionales - si no hubieran tenido la libertad y la 
oportunidad de lograr una movilidad social ascendente? Quizás no. Lo importante aquí es resaltar que las cosas para estos seis jóvenes han cambiado a lo largo del tiempo y gracias a la educación. Tomar nota de ello puede ser la clave para replicar este tipo de estudios con poblaciones más extensas, sin dejar de combinar variables objetivas con subjetivas para comprender mejor cómo ocurren los procesos de movilidad social en México.

\section{Conclusiones}

Este artículo sostiene que la dimensión subjetiva (percepción) es central para comprender la acción de los individuos que se mueven en pos de mejores estadios de desarrollo. Combinadas las percepciones con las variables objetivas, se pueden analizar mejor los patrones de movilidad social.

La exploración de la libertad (capability) que los egresados universitarios perciben que poseen en tres tiempos distintos, muestra racionalidades diversas y contingentes de acuerdo con su contexto específico. Se asocia, por ejemplo, un mayor grado de libertad cuando los profesionales universitarios eran estudiantes, dado el bajo número de responsabilidades. Sin embargo, también asocian la calidad de vida con la libertad real de elegir entre o tener "estabilidad" en su vida cotidiana, u ocupar un puesto laboral demandante y ganar mayores ingresos. No es claro cómo esta "racionalidad razonada" del joven entraría en un modelo clásico de movilidad social ascendente.

Este análisis mostró que, así como las condiciones reales de desarrollo cambiaron a lo largo del tiempo, también lo hizo la subjetividad y con ella, la capacidad de intervención de los jóvenes en sus propios contextos. Al cumplir con sus expectativas educativas, laborales y económicas ("movilidad objetiva"), mantienen una posición más libre en relación con el desarrollo de ellos mismos y de sus hijos. Por ejemplo, desean que sus descendientes elijan lo que ellos mismos valoren y tengan razón de valorar (Sen, 1999) en términos educativos, cuestión que los entrevistados no experimentaron.

La percepción y, por tanto, las normas y las acciones de los seis egresados encuestados para este estudio, cambiaron a lo largo del tiempo. Con esto, podríamos esperar mayores inversiones para cultivar las distintas formas de capital (Bourdieu, 1997) en las nuevas generaciones. No obstante, habría que conducir estudios con poblaciones más extensas para constatar, rechazar o complementar los hallazgos aquí reportados. Finalmente, se trata de responder a la pregunta de si la educación superior en México puede (y bajo qué condiciones) ser un motor real de movilidad social y no solamente una reproductora de desigualdad y cemento de la estratificación social. 


\section{Referencias bibliográficas}

Baert, P. (1998). Social Theory in the Twentieth Century. Gran Bretaña: Polity.

Blanco, E. Solís, P. y Robles, H. (2014). Caminos desiguales. Trayectorias educativas y laborales de los jóvenes en la Ciudad de México. México: INEE-COLMEX.

Bourdieu, P. (1997). The forms of capital. En A. Halsey, H. Lauder, P. Brown, H. Lauder, A. Wells (Eds.), Education: Culture, Economy and Society (pp. 241-258). Oxford: Oxford University Press.

Bryman, A. (2001). Social research methods. Oxford: Oxford University Press.

Cortés, F., Escobar, A. y Solís, P. (2007). Cambio estructural y movilidad social en México. México: COLMEX.

Durán, I. y Soloaga, I. (2015). Percepciones y movilidad social en México. En. R. Vélez, J. Huerta y R. Campos (Eds.), México, ¿el motor inmóvil? (pp. 127-192). México: Centro de Estudios Espinosa Yglesias.

Duru-Bellat, M. y Kieffer, A. (2008). Objective/subjective: the two facets of social mobility. Sociologie du travail, 50S, e1-e18.

Flores-Crespo, P. (2005), Educación superior y desarrollo humano. El caso de tres universidades tecnológicas. México: UIA-ANUIES.

Flores-Crespo, P. (2009), Trayectoria del modelo de universidades tecnológicas en México (1991-2009). Cuadernos de Trabajo de la Dirección General de Evaluación Institucional (DGEI), México: UNAM.

Flores-Crespo, P. y Mendoza, D. (2013), La Educación Superior Tecnológica. El Caso Mexicano, en Incluir a los jóvenes. Retos para la educación terciaria técnica en América Latina, París: IIPE-UNESCO.

Flores-Crespo, P. y Rodríguez Arias, N. (en prensa). Educación superior tecnológica y movilidad social. Un estudio longitudinal basado en historias de vida. Revista Iberoamericana de Educación Superior.

Goldthorpe, J. (1996). Class analysis and the reorientation of class theory: The case of persisting differentials in education attainment. British Journal of Sociology, 473(3), 481-505.

Hansen, M. (2008). Rational action theory and educational attainment. Changes in the impact of economic resources. European Sociological Review, 24(1), 1-17. 
Hernández Sampieri, R., Fernández Collado, C. y Baptista Lucio, P. (2008). Metodología de la investigación. México: McGraw-Hill.

Janesick, V. (2013). Oral history, life history and biography. En A. Trainor, y E. Graue (Eds.), Reviewing qualitative research in the social sciences (pp. 151-165). Nueva YorkLondres: Routledge.

Jaramillo, M. (2016). Medición de bienestar subjetivo y objetivo: ¿complemento o sustituto? Acta Sociológica, 70, 49-71.

Kelly, S. y Kelly, C. (2009). Subjective social mobility: data from 30 nations. En M. Haller, R. Jowell y T. Smith (Eds.), The International Social Survey Programme 1984-2009: Charting the Globe (pp. 128-146). London: Routledge.

Lopreato, J. y Hazelrigg, L. (1970). Intragenerational versus intergenerational mobility in relation to sociopolitical attitudes. Social Forces, 49(2), 200-210.

Nussbaum, M. (2000). Women and Human Development. The Capabilities Approach. Cambridge: Cambridge University Press.

Palomar Lever, J. y Lanzagorta Piñol, N. (2005). Pobreza, recursos psicológicos y movilidad social. Revista Latinoamericana de Psicología, 37(1), 9-45.

Pigou, A. (1932). The economics of welfare (4ta. Ed.). Londres: Macmillan and Co.

Plewis, I. y Bartley, M. (2014). Intragenerational social mobility and educational qualifications. Research in Social Stratification and Mobility, 36, 1-11.

Robeyns, I. (2014). Capabilities y teorías de la justicia social. En M. Nebel, P. Flores-

Crespo, y M. T. Herrera (Cords.), Desarrollo como libertad en América Latina:

Fundamentos y aplicaciones (pp. 73-95). México: UIA.

Rubio, J. (2006). La política educativa y la educación superior en México 1995-2006. Un balance. México: Fondo de Cultura Económica.

Salazar, P., Oliveros, M., Valdez, B. y Coronado, M. (2019). Movilidad social en los graduados de Ingeniería de la Universidad Politécnica de Baja California. Revista de Estudios y Experiencias en Educación, 18(38), 87-111.

Sen, A. (1985). Commodities and capabilities, Holanda: Elsevier Sciencie Publishers.

Sen, A. (1987). The standard of living. Cambridge: Cambridge University Press.

Sen, A. (1999). Development as freedom. New York: Anchor Books.

Sen, A. (2009). The idea of justice. Londres: Allen Lane. 
Solís, P. (2012). Social mobility in Mexico. Trends, recent findings and research challenges. Trace. Travaux et recherches dans les Amériques du Centre, (62), 7-20.

Stocké, V. (2007). Explaining educational decision and effects of families social, social class position. An empirical test of the Breen-Goldthorpe model of educational attainment. European Sociological Review, 23(4), 505-519.

Van den Berg, M. (2011). Subjective social mobility: Definitions and expectations of 'moving up' of poor Moroccan women in the Netherlands. International Sociology, 26(4) $503-523$

Vélez Grajales, R., Campos Vázquez, R. M. y Fonseca, C. E. (2015). El concepto de movilidad social: Dimensiones, medición y estudios en México. Centro de Estudios Espinosa Yglesias, Documento de trabajo No. 001/2015

Villa Lever, L. (2016). Educación superior, movilidad social y desigualdades interdependientes. Universidades, 68,51-64

Westoff, C., Bressler, M. y Sagi, P. (1960). The Concept of Social Mobility: An Empirical Inquiry. American Sociological Review, 25(3), 375-385. 